\title{
Modernization of TRR Control Rod Mechanisms Driving System Based on FPGA
}

\author{
Mohammad Arkani ${ }^{1,2}$ \\ ${ }^{1}$ Physics Department \\ Amir-Kabir University of Technology \\ Hafez Avenue Tehran, Iran. \\ ${ }^{2}$ Radiation Application Research School \\ Nuclear Science \& Technology Research Institute \\ (NSRTI) Atomic Energy Organization of Iran (AEOI) \\ e-mail:arkani_m@yahoo.com
}

\author{
Hossein Khalafi, Ashkan Hosnirokh, Arsaln Ezati \\ Radiation Application Research School \\ Nuclear Science \& Technology Research Institute \\ (NSRTI) Atomic Energy Organization of Iran (AEOI)
}

\begin{abstract}
Research reactors are important neutron sources for research and radioisotope production purposes. The common problem of these facilities is their obsolete instrumentation and control (I\&C) systems. The spare parts are not still available as their production is discontinued; therefore, the maintenance is a difficult problem. A major attitude of aging management of research reactors is modernization of the old age I\&C systems. In this work, a modern control rod driving system for TRR control rod mechanisms is designed and constructed using state of the art electronic technology. Unlike the original analog system, selfcheck capability and essential interlocks are implemented. The system is cost effective as FPGA technology is utilized. Future upgrades are possible as FPGA is a reprogrammable device. The proposed equipment is recommended to be installed in other nuclear research reactors, which suffer from aged I\&C systems.
\end{abstract}

Keywords-Field Programmable Gate Array (FPGA); Instrumentation and Control System, Nuclear Reactor, Control Rod Mechanism.

\section{NOMENCLATURE}

ADC: Analog-to-Digital Converter CFE: Control Fuel Element CRMDS: Control Rod Mechanisms Driving System CRM: Control Rod Mechanism

DCS: Distributed Control System

FPGA: Field Programmable Gate Array

HDL: Hardware Description Language

IAEA: International Atomic Energy Agency

I\&C: Instrumentation and Control

MOSFET: Metal Oxide Field Effect Transistor

RPCRS: Reactor Power Control and Regulating System RPS: Reactor Protection System

RR: Regulating Rod

SR: Safety Rod

TRR: Tehran Research Reactor

\section{INTRODUCTION}

Majority of operating research reactors has been put into operation since 1950. Modifications, upgrading and refurbishing have been taken by the operating and maintenance personal to meet the requirements for better performance and reliability. However, many of these old research reactors are still operating with their original I\&C systems. As I\&C systems are important for reactor safety to protect against abnormal conditions, modernization and aging management of these reactors are recommended by IAEA [1]. Out-dated I\&C systems cause operational problems as well as difficulties in obtaining replacement parts. In addition, satisfying the stringent safety conditions laid out by the nuclear regulatory bodies requires the modernization of research reactors I\&C systems and integration of additional instrumentation units to the reactor. Technical advances in I\&C systems have been rapid in the past decade. The demands of high level sophistication and reliability to meet various operational and safety requirements are being met by the increased use of modern technologies. This requires careful consideration of the research reactor operators in planning how to improve the I\&C systems [2-4].

The technology of I\&C systems of nuclear reactors has, namely, undergone evolution for decades from vacuum tubes and transistors to digital technology. The demands of high-level sophistication and reliability are being met by the increased use of digital technologies. Self-test features and on-line diagnostic capabilities of digital systems to monitor any degradation in performance of I\&C systems are unique features of the technology. In addition, testability and maintainability are perfectly possible.

I\&C systems of nuclear research reactors need to be upgraded in a reliable and cost-effective manner to replace obsolete equipment, to reduce operation and maintenance costs, to improve plant performance, and to enhance safety. Technological improvements, particularly the availability of digital systems, offer improved functionality, performance, and reliability; solutions to obsolescence of analog equipment; reduction in operation and maintenance costs; and the potential to enhance safety. Modern digital technology holds a significant potential to improve costeffectiveness and productivity of nuclear reactors.

\section{TRR CRMS}

Most research reactors are controlled by the insertion of neutron absorbers (control rods) into the reactor core. Control rods require linear movement mechanisms, which should be fail-safe, reliable, fast acting and radiation resistant. A control rod drive mechanism basically consists 
of a drive motor coupled through gearing, an electromagnetic holding device to the neutron absorber, a few limit switches, and position measuring devices. On a scram signal, the electromagnet is de-energized by RPS, therefore allowing the control element to be inserted into the core under gravity to inject negative reactivity. The movement of the neutron absorber is suitably damped by shock absorbers at the end of travel to prevent mechanical damage to the absorber, to the control fuel assembly, and to the mechanism. Fig. 1 shows a schematic view of a TRR control rod mechanism. The pneumatic shock absorber is firstly activated to reduce the speed of the control rod. Then a mechanical dashpot stops the control rod.

TRR core consists of four SRs and a single RR. The compensation of excess reactivity and its changes during reactor operation is made by SRs. The power adjustment under the automatic and manual control is possible by RR. As the total reactivity worth of this absorber is lower than one dollar, avoiding prompt criticality is guaranteed. The total reactivity worth of each SR is around three dollars.

A step motor and a compound system of nut and screw make the linear axial movement possible. A ten-turn potentiometer connected to the nut and screw system measures the position of the electromagnet, while three limit switches detect the two extreme positions of the mechanism. The contact between magnet (mechanism) and the control rod is monitored by a contact-switch. From lower limit to the upper limit, the distance is equal to $64 \mathrm{~cm}$. Regarding the reactivity insertion limit in pool type research reactors [5], seven minutes is taken by SRs to move from lower limit to the upper limit. This time is variable for RR between three minutes and seven minutes.

\section{ORIGINAL TRR CRMDS}

Fig. 2 shows the original TRR mechanisms driving system installed on the reactor bridge. This system has three major parts as shown on the figure:

- Power supply unit

- Cable connections: Each mechanism has a cable connection to the driver. The driver sends the status

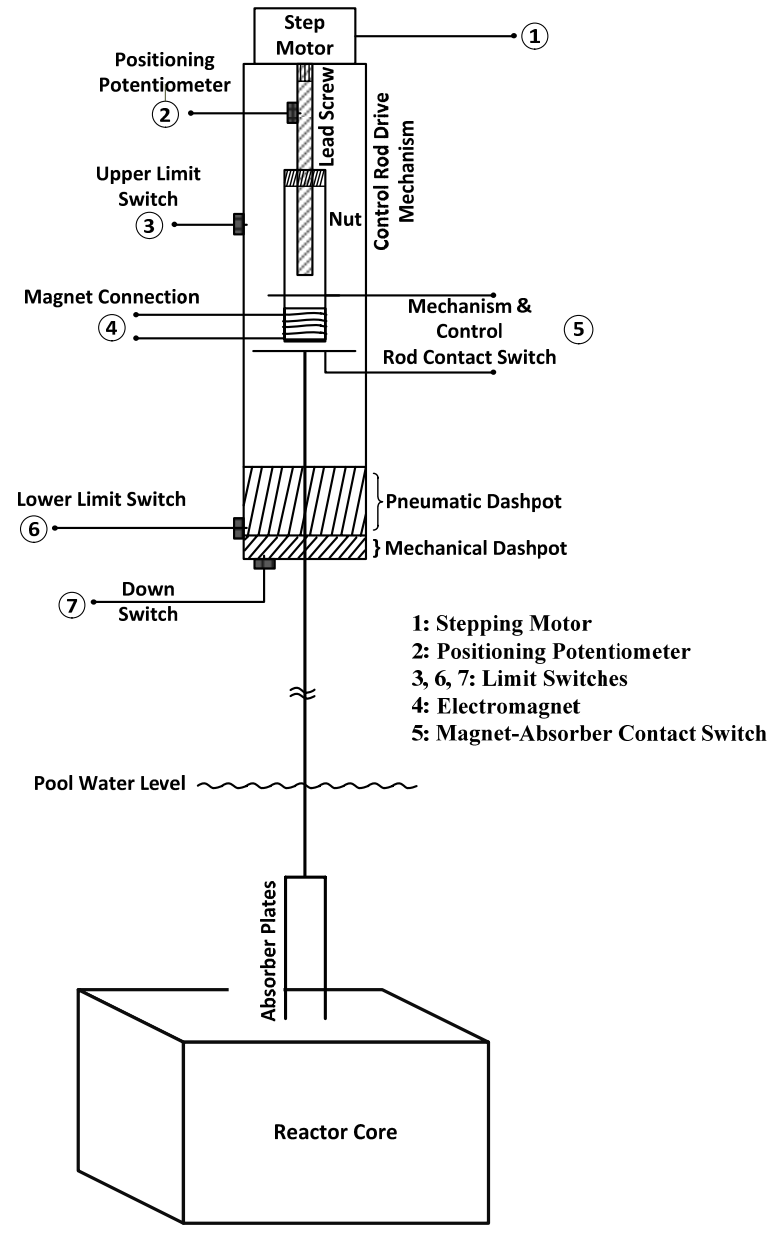

Figure 1. Schematic diagram of a TRR CRM

signals to the reactor console and receives the commands. The reactor utilizes five mechanisms; therefore, there are ten cable connections.

- Driver modules: SR1 and SR2 has a specific module as same as SR3 and SR4. The RR has a especial driver module, as it is more complex due to the speed control function. Under automatic reactor control, depending on the reactor period and difference between reactor power and power demand, the RPCRS trims the RR movement speed. Soft and optimized regulation of the reactor power is achieved by automatic reactor control. Therefore, speed control function is an important feature of RR driver.

Some bullet points of the original TRR CRMDS can be listed as follows [6, 7, 8]: 


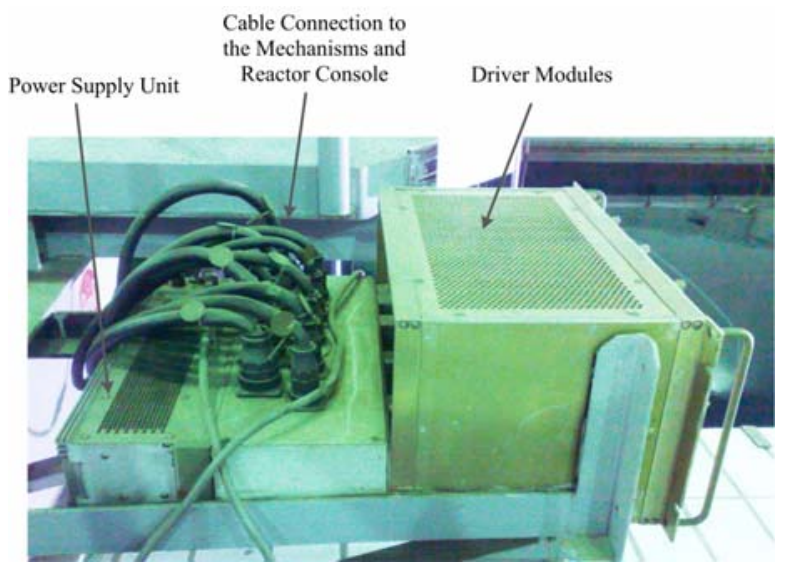

Figure 2. Original TRR CRMDS, which is installed on the reactor bridge.

- Status and command signals of the mechanisms are connected to the reactor console using hardwire connection. Isolated status signals of the mechanisms (positioner, upper limit switch, lower limit switch, . . .) are essential input signals for future extensions of TRR I\&C systems. These signals are not available by the original system.

- An internal RC oscillator determines movement speed of each absorber. This is an analog design. Therefore a routinely calibration program should be taken. Note that a voltage to frequency converter performs time discretization in RR driver unit. The function block diagram of the original driver unit will be presented shortly.

- Self-check function is one of safety advantages of digital technology, which is not provided by the original system.

- The architecture, electronic components, and standards are obsolete. Therefore, maintenance of the system and renewing of the units are difficult.

- Due to the aging of the system, the safety is degraded.

Fig. 3 shows a simplified block diagram of the original TRR CRMDS. The movement speed control input is conditioned and converted to frequency. The appropriate logic state is generated based on input commands and mechanism status. The control unit handles the current amplifiers to energize the stepping motor.

I\&C systems of TRR is upgrading now. Process instruments and nuclear instruments are going to be replaced with modern state of the art technology. DCS control strategy will be employed for monitoring and control of the reactor. Therefore, incoming changes in the structure of the I\&C systems enforce new compatible technology.

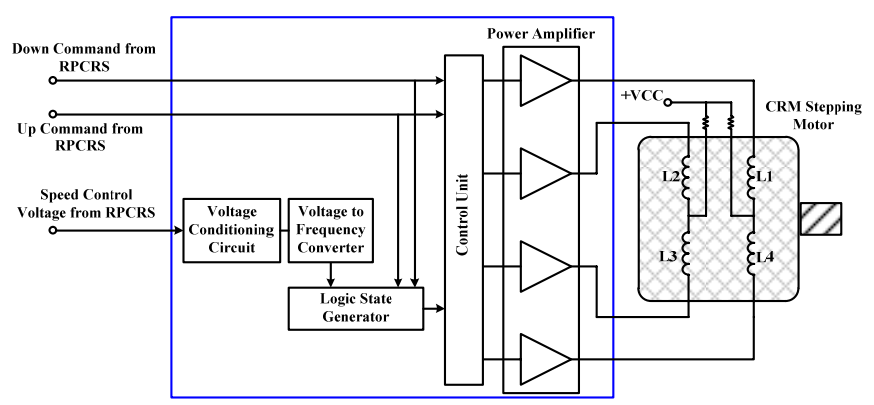

Figure 3. Original TRR CRMDS functional block diagram.

\section{MODERN INSTRUMENTATION AND FPGA TECHNOLOGY}

FPGAs are among the most rapidly growing processing platforms for high-performance processing applications. As FPGA technology has grown in both complexity and sophistication, its uses have spread. Simple physical design circuitry, capability of revising of the design based on programming, and compactness are important advantages of the technology. Unlike processors, FPGAs are truly parallel in nature; hence, different processing operations do not have to compete for the same hardware resources. Each independent processing task is assigned to a dedicated section of the chip, and can function autonomously without any influence from other logic blocks. As a result, performance of one part of the application is not affected when more processing tasks are added. Controlling inputs and outputs (I/O) at the hardware level provides more fast response time and specialized functionality to closely match application requirements. FPGA technology offers flexibility and rapid prototyping. The physical nature of programmable silicon means no fabrication costs or no long lead times for assembling. System requirements often change over the time, the cost of making changes to FPGA designs is negligible. Being reconfigurable, FPGA chips can keep up with future modifications that might be necessary. The compactness is an important advantage of FPGA. As the technology offers an effective solution to digital implementations, extensive research has been conducted on modernization of traditional instruments [e.g. 9].

\section{NEW TRR CRMDS}

Based on the experiences gained by operation and maintenance of TRR during years of utilization, and to response the future needs of the I\&C upgrading program and safety requirements, a proper CRMDS is designed and 


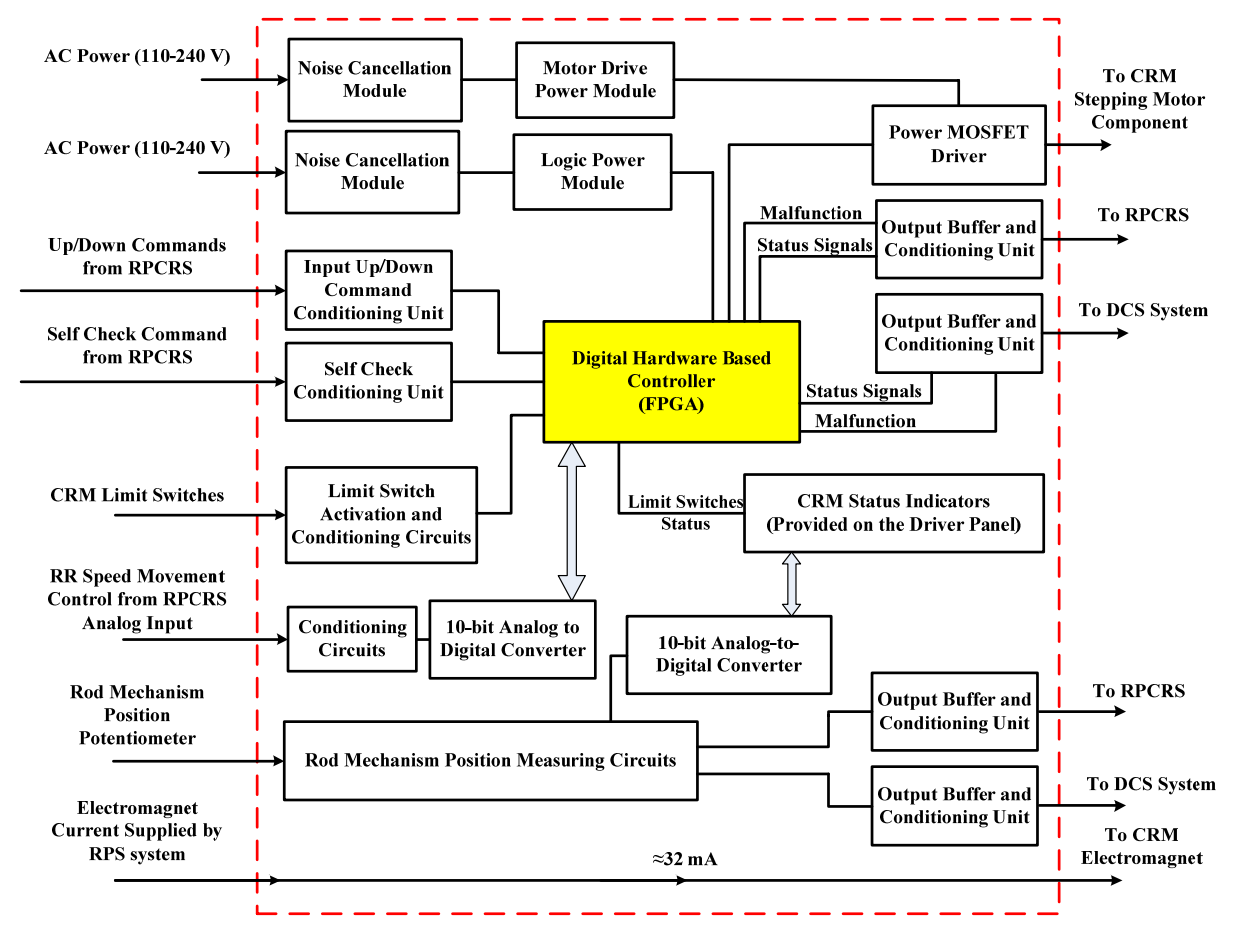

Figure 4. Functional block diagram of the new TRR CRMDS

constructed. Fig. 4 illustrates the function block diagram of the system. The main part of this system is the reprogrammable FPGA-based hardware. All inputs and outputs of the system are controlled by this block. The safety interlocks ${ }^{1}$, self-check functions, absorber movement speed control functions, and management of status signals are implemented into the FPGA. Any communication between input/output ports needs an appropriate voltage standard conditioning/conversion circuit as shown in Fig. 4.

The control of absorber movement speed is performed by an analog input signal. The 10-bit ADC converts the input signal to an integer format number. FPGA controls the ADC timing and communicates the data. The integer number, which is provided by the ADC, is processed by the FPGA to produce appropriate triggering clock pulses to run the stepping motor of the mechanism. This is performed by HDL programming. Fig. 5 shows a simple illustration of the stepping motor clock generator. System clock is the input of the PLL to produce the appropriate digitization frequency, which is fed into the up counter block. When the counter output goes greater than $\mathrm{Nc}^{2}$, the 16-bit integer number, the comparator output is set by the next rising edge of the system clock. Then, the up counter is reset and the process

\footnotetext{
${ }^{1}$ Safety interlocks are the logics that protect mechanisms against unsafe conditions, which may cause mechanical damage.

${ }^{2} \mathrm{Nc}=($ ADC Output $\times \mathrm{K} 1)+\mathrm{K} 2$
}

is repeated. $\mathrm{K} 1$ and $\mathrm{K} 2$ are constants, which depend on the electromechanical characteristics of the reactor CRM. Note that zero input voltage of the ADC means minimum speed ${ }^{3}$ of the absorber, which is determined by K2. The system utilizes two separate power modules for logic circuits and Power MOSFET Driver block. This is necessary for noise immunity against electromagnetic effects of the stepping motor operation. Note that optical isolation between motor driver and FPGA is provided. Each unit of the driver has a panel of status indicators for limit switches, RR speed, control rod position, and upward or downward movements.

All signals are buffered and conditioned appropriately. The control and management of all inputs and outputs is performed by the FPGA except electromagnet current. This is supplied by RPS, which is connected to the mechanisms through the same cable connection. As the driver is a safety related system, this function should not be controlled by the driving system. Figs 6 to 8 show the prototypes of the new TRR CRMDS. Each couple of SRs mechanisms has a specific system unit while RR has a dedicated individual module. Details are illustrated on the figures.

\footnotetext{
${ }^{3}$ Details about TRR CRM were explained in the previous sections.
} 


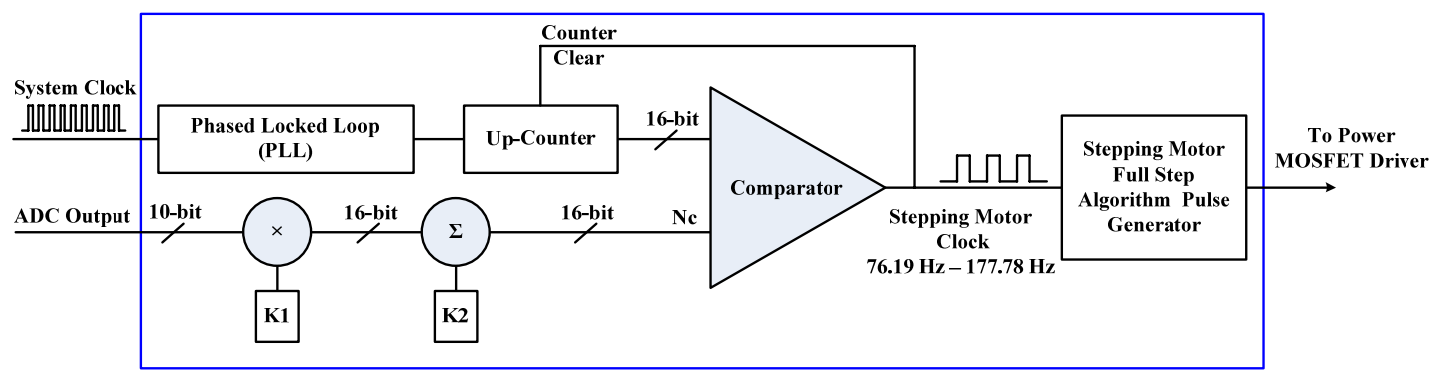

Figure 5. Functional block diagram of the HDL program implemented in the FPGA for speed control of CRM stepping motor

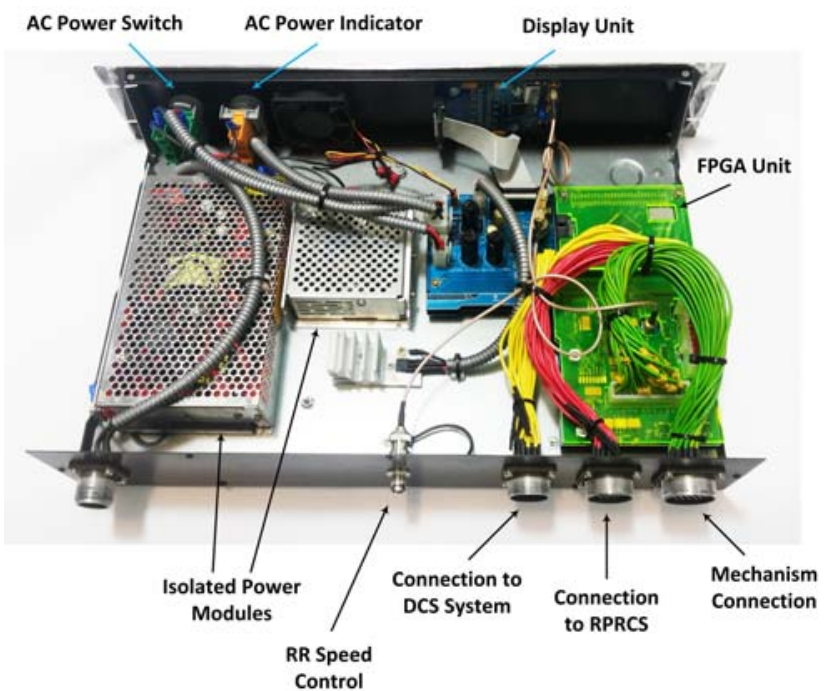

Figure 6. RR driver module, top and rear view

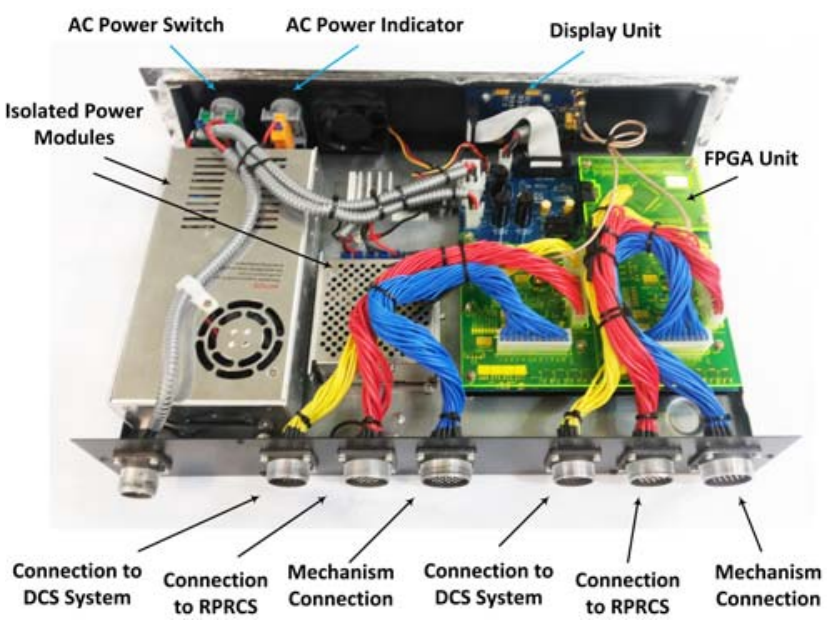

Figure 7. SR1 and SR2 driver module, top and rear view

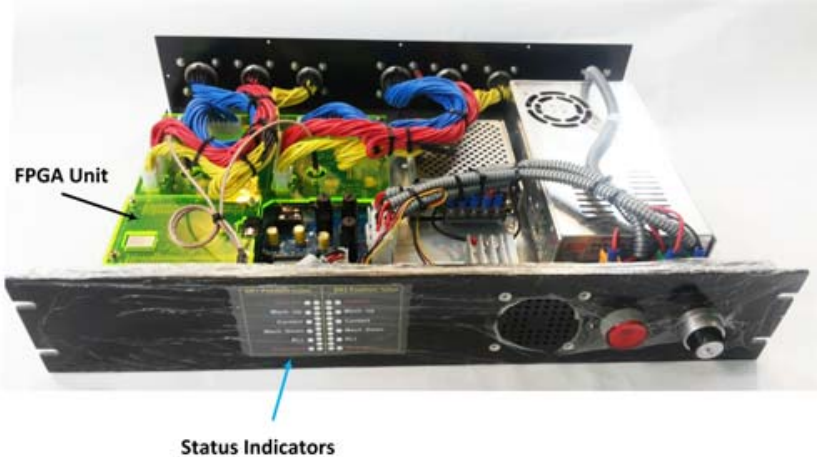

Figure 8. SR1 and SR2 driver module, top and front view

The CRMDS was tested for 42 hours of continuous operation. The test was performed on the $\mathrm{RR}^{4}$ mechanism in an oscillatory mode. No defects were seen in the system during the experiment.

Table I shows the timing test results of TRR CRMDS. The original and new systems are compared. As the digital technology is employed, the new system show a uniform behavior for all CRMs. The maximum error is limited to $1.9 \%$. This is different for the original driving system. Each mechanism shows a different behavior due to the analog structure. Especially for RR, the error is considerable in both manual and automatic modes. The results show a considerable difference between SAR set points for CRM drivers and its experimental test results for the original parts. Unfortunately, no calibration or checking program for the original system is codified in TRR during years of reactor operation. Therefore, the control system of TRR has been working in an unknown condition. This is an important drawback of analog systems which need a routinely test and calibration program.

\footnotetext{
${ }^{4}$ The electromagnet was not energized during the experiment.
} 
TABLE I. Test results of TRR CRMDS.

\begin{tabular}{|c|c|c|c|c|}
\hline \multirow{2}{*}{ CRMDS } & Mechanism & $\begin{array}{c}\text { SAR }^{\mathbf{a}} \\
\mathbf{m : s s}\end{array}$ & $\begin{array}{c}\text { Measured } \\
\mathbf{m}: \mathbf{s}\end{array}$ & Error\% \\
\hline \multirow{2}{*}{ New System } & Regulating Rod & $3: 00 \sim 7: 00$ & $3: 03 \sim 6: 52$ & $-1.67 \sim-1.9$ \\
\cline { 2 - 5 } & Safety Rod 1 4 & $7: 00$ & $6: 52$ & -1.9 \\
& & & Manual: $2: 06$ & Manual: -30 \\
& Regulating Rod & $3: 00 \sim 7: 00$ & Automatic: $1: 37$ & Automatic: -46.1 \\
\cline { 2 - 5 } Original & & & SR1: $7: 14$ & SR1: 3.3 \\
& & & SR2: $7: 15$ & SR2: 3.6 \\
& System & & SR3: $7: 09$ & SR3: 2.1 \\
& Safety Rod 1 4 & $7: 00$ & SR4: $7: 10$ & SR4: 2.4 \\
\hline
\end{tabular}

a Safety Analysis Report for Tehran Research Reactor [10]

${ }^{\mathrm{b}} \mathrm{RR}$ tests are performed under below conditions:

Movement range: $3 \%$ up to $\% 100$

Power demand in automatic mode $=5 \mathrm{MW}$

Reactor status during experiment: Subcritical

\section{CONCLUSION}

A very common problem among operating nuclear research reactors is the aging management of the I\&C systems. The technology used in the original I\&C systems are obsolete, therefore maintenance is an important problem. The general attitude is modernization of aging parts of the system with the replacement priority. In TRR, modernization and refurbishment is now in progress. Accordingly, TRR CRMDS was studied to be replaced with state of the art technology. A digital system based on FPGA was designed and constructed. The prototypes of the driver showed acceptable results. Self-check and protection interlocks was implemented in the system. The equipment is suitable for the specific requirements in TRR. As FPGA is a reprogrammable device, future upgrades are easily possible. The design concepts are recommended for other aging nuclear research reactors.

\section{REFERENCES}

[1] IAEA-TECDOC-973, Research reactor instrumentation and control technology, International Atomic Energy Agency, Report of a Technical Committee meeting held in Ljubljana, 48 December 1995.

[2] H. Schulz, Challenges left in the areas of materials ageing and plant modernization, Nuclear Engineering and Design, Volume 209, Issues 1-3, November 2001, Pages 57-65.

[3] IAEA-TECDOC-1016, Modernization of instrumentation and control in nuclear power plants, INTERNATIONAL ATOMIC ENERGY AGENCY, May 1998.

[4] IAEA-TECDOC-1625, Research Reactor Modernization and Refurbishment, International Atomic Energy Agency, August 2009.

[5] H. Kazeminejad, Reactivity insertion limits in a typical pooltype research reactor cooled by natural circulation, Annals of Nuclear Energy, Volume 33, Issue 3, February 2006, Pages 252-261.

[6] Technical Specifications of TRR Safety Control Rod Drive Mechanisms, INVAP Manual, Invap-Man-021.

[7] Stepping Motor Controller for TRR Regulating Rod, INVAP Manual, Invap-Man-026.

[8] Stepping Motor Controller for TRR Shim Safety Rods, INVAP Manual, Invap-Man-013.

[9] Implementing Digital Instrumentation and Control Systems in the Modernization of Nuclear Power Plants, IAEA Nuclear Energy Series, No. NP-T-1.4, April 2009.

[10] Safety Analysis Report for Tehran Research Reactor, October 2007, Atomic Energy Organization Of Iran. 